\title{
Cbl-b predicts postoperative survival in patients with resectable pancreatic ductal adenocarcinoma
}

\author{
Qian Dong1, Yuteng Ma², Yao Zhang ${ }^{3}$, Xiujuan Qu ${ }^{4}$, Zhi Li ${ }^{4}$, Yafei Qi ${ }^{5}$, Yunpeng Liu ${ }^{4}$, \\ Ce $\mathrm{Li}^{4}$, Kai $\mathrm{Li}^{1}$, Xianghong Yang ${ }^{5}$ and Xiaofang $\mathrm{Che}^{4}$ \\ ${ }^{1}$ Department of Oncology, Shengjing Hospital of China Medical University, Shenyang 110004, China \\ ${ }^{2}$ Department of Gastrointestinal Surgery, Peking University Cancer Hospital, Beijing 100142, China \\ ${ }^{3}$ Department of Ultrasound, Shengjing Hospital of China Medical University, Shenyang 110004, China \\ ${ }^{4}$ Department of Medical Oncology, The First Hospital of China Medical University, Shenyang 110001, China \\ ${ }^{5}$ Department of Pathology, Shengjing Hospital of China Medical University, Shenyang 110004, China \\ Correspondence to: Xianghong Yang, email: xhyang4933@vip.sina.com \\ Xiaofang Che, email: chexf_2015@126.com \\ Keywords: pancreatic ductal adenocarcinoma (PDAC), Cbl-b, prognosis \\ Received: January 01, $2017 \quad$ Accepted: May 21, $2017 \quad$ Published: June 27, 2017 \\ Copyright: Dong et al. This is an open-access article distributed under the terms of the Creative Commons Attribution License 3.0 \\ (CC BY 3.0), which permits unrestricted use, distribution, and reproduction in any medium, provided the original author and source \\ are credited.
}

\section{ABSTRACT}

Casitas B-lineage lymphoma b (Cbl-b) is a ubiquitin-protein ligase and a signal transducing adaptor protein involved in immune regulation, and it may be involved in the development and progression of cancer. We investigated the association between Cbl-b expression and prognosis in patients with resectable pancreatic ductal adenocarcinoma (PDAC). The clinicopathological characteristics and survival data of 134 patients with surgery for PDAC between January 2009 and February 2012 were retrospectively evaluated, and Cbl-b expression was assayed by immunohistochemical staining. The association of $\mathrm{Cbl}-\mathrm{b}$ expression with clinicopathological features and postoperative prognosis was analyzed. Cbl-b expression was strongly associated with the pathological primary tumor $(\mathrm{pT})$ category $(P=0.005)$ and pathological TNM (pTNM) stage $(P=0.035)$, but not with other clinicopathological characteristics (all $P>0.05)$. In addition to current markers including pathological regional lymph nodes (pN) category, CA19-9, and histological differentiation, univariate and multivariate analysis found that Cbl-b was independently associated with overall survival (OS) of patients with resectable PDAC. Cbl-b was predictive of OS in a subgroup of patients with serum CA19-9 $\geq 37 \mathrm{U} / \mathrm{mL}$. Cbl-b expression combined with pN, histological differentiation, and CA19-9 level could be used as a novel clinical model predictive of OS for patients with resectable PDAC. In conclusion, $\mathrm{Cbl-b}$ in resectable PDAC was an independent predictor of adverse prognosis. Cbl-b expression together with pN, histological differentiation, and CA19-9 level might lead to improved risk stratification and prognosis for patients with resectable PDAC.

\section{INTRODUCTION}

Despite advances in treatment, the prognosis of pancreatic cancer is dismal, and the 5-year survival rate is $<7 \%$ [1]. In China, the cancer statistics show that the 5 -year survival rate of pancreatic cancer is $4.1 \%$ and the median survival time is only 3.9 months [2]. For patients with pancreatic cancer, surgical resection is the only curative treatment, but fewer than $20 \%$ of patients are indicated for radical surgery, and their 5-year survival rate is only $10 \%-25 \%[3,4]$. It is very important to accurately predict the prognosis after tumor resection for 
the assessment of therapeutic effect, choice of adjuvant therapy, and informing patients.

To date, various biomarkers have been reported to be the prognostic markers for pancreatic cancer. Among them, the serum carbohydrate antigen 19-9 (CA19-9) level is the most widely accepted tumor marker for pancreatic cancer. It has been reported that the CA19-9 level can be used in the diagnosis, the evaluation of resectability, monitoring the progression and the prognostic prediction of pancreatic cancer [5-7]. In addition, some new prognostic markers, such as PD1/PD-L1 [8], non-coding RNAs [9] and circulating tumor DNA [10], have been reported recently. However, it may be more meaningful to identify prognostic markers from molecules involved in the malignant biological behavior of pancreatic cancer. Despite being a hypovascular malignant tumor, pancreatic cancer cells are still capable of rapid proliferation. The strong proliferative capacity significantly affects the prognosis of patients. Casitas B-lineage lymphoma b (Cbl-b) is a member of the ubiquitin ligase casitas B (Cbl) family and acts as a ubiquitin-protein ligase and adaptor protein [11, 12]. We previously showed that silencing Cbl-b expression activated the Smad3/p21 axis and inhibited proliferation of pancreatic ductal adenocarcinoma (PDAC) cells [9]. However, whether Cbl-b can be used as a prognostic marker for pancreatic cancer remains unknown. So far, studies of the association between Cbl-b and prognosis in malignancies are rare[13-15]. Li et al reported that presence of the rs2305035, a variant AA or AG genotypes was associated with overall survival (OS) [16], and patients with $\mathrm{Cbl}$ family mutations (e.g., c-Cbl, Cbl-b, and (Cbl-c) for myeloid malignancies had poor prognosis [17]. However, there is no relevant research about the prognostic value of Cbl-b expression in PDAC patients. If a relationship between Cbl-b expression and the prognosis in PDAC does exist, then interfering with Cbl-b expression or the Cbl-b signal pathway may be able to prolong survival of PDAC patients, and thus shed light on potential therapeutic targets and prognostic biomarkers.
The study aim was to determine the prognostic value of Cbl-b in patients with PDAC. The expression of Cbl-b in human PDAC tissues was evaluated by immunohistochemistry, and its association with clinical outcomes was evaluated. The results may improve our understanding of the clinical significance of Cbl-b in PDAC and its prognostic value in PDAC patients.

\section{RESULTS}

\section{Demographic and clinicopathological characteristics}

The demographic and clinical characteristics of the 134 included patients are shown in Table 1. The median age was 60 years $\left(\mathrm{P}_{25}-\mathrm{P}_{75}, 54-68\right.$ years; range, 35-80 years), and $78(58.2 \%)$ were men. The median tumor size was $4.0 \mathrm{~cm}$ (range, $1.2-10 \mathrm{~cm}$ ), 113 patients $(84.3 \%)$ had well or moderately differentiated tumors, 21 $(15.7 \%)$ had poorly differentiated tumors, $130(97.0 \%)$ had negative surgical margins, and 42 (31.3\%) had lymph node metastasis. Sixty-nine $(51.5 \%)$ patients had tumors that extended beyond the pancreas. $3(2.2 \%)$ had vascular tumor thrombi, and $33(24.6 \%)$ had invasion of adjacent organs. At the last follow-up, 112 patients $(83.6 \%)$ had died. Median OS was 15.9 months [95\% confidence interval (CI): 13.0-18.7] (Supplementary Figure 1).

\section{Association of Cbl-b expression and clinicopathological characteristics}

Cbl-b protein expression was assayed by immunohistochemistry, and positively stained cells were identified by the presence of brown-yellow particles located in the membrane and cytoplasm (Figure 1). Cbl-b expression in PDAC tissues varied, and was strongly associated with pT $(P=0.005)$ and pTNM stage $(P=$ 0.035 ), but was not correlated (all $P>0.05$ ) with other clinicopathological characteristics (Table 1).

\section{A Cbl-b high expression}

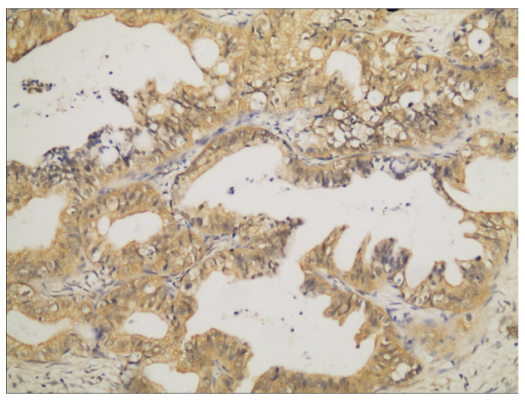

\section{B Cbl-b low expression}

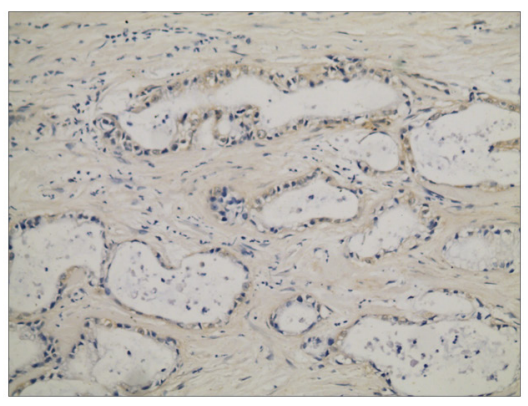

Figure 1: Cbl-b expression in PDAC tissue assayed by immunohistochemistry. Positive expression is shown by brown-yellow particles distributed in the cell membrane and cytoplasm (SP staining $\times 200$ ). (A) Strong Cbl-b high expression. (B) Weak Cbl-b expression. 
Table 1: The relationship of Cbl-b expression and clinicopathological characteristics in 134 patients with resectable pancreatic ductal adenocarcinoma

\begin{tabular}{|c|c|c|c|c|c|}
\hline \multirow[t]{2}{*}{ Characteristics } & \multirow{2}{*}{$\begin{array}{l}\text { No. of cases } \\
\quad(n, \%)\end{array}$} & \multicolumn{2}{|c|}{ Cbl-b expression } & \multirow[t]{2}{*}{$\chi^{2}$} & \multirow[t]{2}{*}{$P$ value } \\
\hline & & Low & High & & \\
\hline \multicolumn{6}{|l|}{ Age (years) ${ }^{*}$} \\
\hline$<60$ & $65(48.5)$ & $18(40.9)$ & $47(52.2)$ & 1.514 & 0.218 \\
\hline$\geq 60$ & $69(51.5)$ & $26(59.1)$ & $43(47.8)$ & & \\
\hline \multicolumn{6}{|l|}{ Sex } \\
\hline Male & $78(58.2)$ & $30(68.2)$ & $48(53.3)$ & 2.678 & 0.102 \\
\hline Female & $56(41.8)$ & $14(31.8)$ & $42(46.7)$ & & \\
\hline \multicolumn{6}{|c|}{ Maximum tumor diameter $(\mathrm{cm})^{\#}$} \\
\hline$<4.0$ & $57(42.5)$ & 17(38.6) & $40(44.4)$ & 0.408 & 0.523 \\
\hline$\geq 4.0$ & $77(57.5)$ & $27(61.4)$ & $50(55.6)$ & & \\
\hline \multicolumn{6}{|l|}{ Differenciation } \\
\hline well/ moderately & $113(84.3)$ & 39 (88.6) & $74(82.2)$ & 0.920 & 0.337 \\
\hline poor & $21(15.7)$ & $5(11.4)$ & $16(17.8)$ & & \\
\hline \multicolumn{6}{|l|}{ Surgical margins } \\
\hline Negative & $130(97.0)$ & $42(95.5)$ & $88(97.8)$ & - & $0.597^{\&}$ \\
\hline Positive & $4(3.0)$ & $2(4.5)$ & $2(2.2)$ & & \\
\hline \multicolumn{6}{|l|}{ pT category } \\
\hline $\mathrm{pT} 1+\mathrm{pT} 2$ & $65(48.5)$ & $29(65.9)$ & $36(40.0)$ & 7.942 & 0.005 \\
\hline $\mathrm{pT} 3+\mathrm{pT} 4$ & $69(51.5)$ & $15(34.1)$ & $54(60.0)$ & & \\
\hline \multicolumn{6}{|l|}{$\mathrm{pN}$ category } \\
\hline $\mathrm{pN} 0$ & $92(68.7)$ & $33(75.0)$ & $59(65.6)$ & 1.225 & 0.268 \\
\hline $\mathrm{pN} 1$ & $42(31.3)$ & $11(25.0)$ & $31(34.4)$ & & \\
\hline \multicolumn{6}{|c|}{ Vascular tumor thrombus } \\
\hline No & $131(97.8)$ & $43(97.7)$ & $88(97.8)$ & - & $1.000^{\&}$ \\
\hline Yes & $3(2.2)$ & $1(2.3)$ & $2(12.2)$ & & \\
\hline \multicolumn{6}{|c|}{ Adjacent organs invasion } \\
\hline No & $101(75.4)$ & $37(84.1)$ & $64(71.1)$ & 2.682 & 0.101 \\
\hline Yes & $33(24.6)$ & $7(15.9)$ & $26(28.9)$ & & \\
\hline \multicolumn{6}{|l|}{ pTNM category } \\
\hline I & $53(39.6)$ & $23(52.3)$ & $30(33.3)$ & 4.434 & 0.035 \\
\hline II /III & $81(60.4)$ & $21(47.7)$ & $60(66.7)$ & & \\
\hline \multicolumn{6}{|l|}{ CA19-9 $(\mathrm{U} / \mathrm{mL})^{* *}$} \\
\hline$<37$ & $18(15.5)$ & $5(13.2)$ & $13(16.7)$ & 0.240 & 0.624 \\
\hline$\geq 37$ & $98(84.5)$ & $33(86.8)$ & $65(83.3)$ & & \\
\hline
\end{tabular}

*Age (years): Median $\left(\mathrm{P}_{25}-\mathrm{P}_{75}\right): 60$ (54-68).

\# Maximum tumor diameter (cm): Median $\left(\mathrm{P}_{25}-\mathrm{P}_{75}\right): 4.0$ (3.0-5.63).

\& Fisher's exact test.

** Of the 134 patients included in the present study, the values of serum CA19-9 were available for 116 patients. There were 18 missing values for CA19-9.

pT: pathological primary tumor; pN: pathological regional lymph nodes; pTNM: pathological TNM. 
Table 2: Univariate analysis of overall survival (OS) and clinicopathological characteristics in 134 patients with resectable pancreatic ductal adenocarcinoma

\begin{tabular}{|c|c|c|c|}
\hline Characteristics & $\begin{array}{c}\text { Median OS (months) } \\
(95 \% \mathrm{CI})\end{array}$ & Log-rank $x^{2}$ & $P$ value \\
\hline \multicolumn{4}{|l|}{ Age (years) } \\
\hline$<60$ & $14.2(11.4-17.0)$ & 3.874 & 0.049 \\
\hline$\geq 60$ & $18.1(9.2-27.0)$ & & \\
\hline \multicolumn{4}{|l|}{ Sex } \\
\hline Male & $15.0(11.3-18.7)$ & 0.327 & 0.568 \\
\hline Female & $17.4(13.6-21.2)$ & & \\
\hline \multicolumn{4}{|c|}{ Maximum tumor diameter $(\mathrm{cm})$} \\
\hline$<4.0$ & $25.3(13.6-37.1)$ & 3.504 & 0.061 \\
\hline$\geq 4.0$ & $13.2(9.7-16.6)$ & & \\
\hline \multicolumn{4}{|l|}{ Differenciation } \\
\hline well/ moderately & $17.2(13.4-21.1)$ & 6.162 & 0.013 \\
\hline poor & $7.8(2.1-13.5)$ & & \\
\hline \multicolumn{4}{|l|}{ Surgical margins } \\
\hline Negative & $15.9(13.3-18.4)$ & 0.324 & 0.569 \\
\hline Positive & $24.9(5.6-44.2)$ & & \\
\hline \multicolumn{4}{|l|}{ pT category } \\
\hline $\mathrm{pT} 1+\mathrm{pT} 2$ & $23.7(14.7-32.8)$ & 3.502 & 0.061 \\
\hline $\mathrm{pT} 3+\mathrm{pT} 4$ & $14.1(11.6-16.7)$ & & \\
\hline \multicolumn{4}{|l|}{ pN category } \\
\hline pN0 & $18.9(14.0-23.8)$ & 6.815 & 0.009 \\
\hline $\mathrm{pN} 1$ & $11.0(7.7-14.4)$ & & \\
\hline \multicolumn{4}{|c|}{ Vascular tumor thrombus } \\
\hline No & $16.2(13.6-18.9)$ & 0.051 & 0.821 \\
\hline Yes & $13.7(0.1-27.4)$ & & \\
\hline \multicolumn{4}{|c|}{ Adjacent organs invasion } \\
\hline No & $16.4(12.7-20.1)$ & 0.686 & 0.408 \\
\hline Yes & $15.1(12.3-17.8)$ & & \\
\hline \multicolumn{4}{|l|}{ pTNM stage } \\
\hline I & $25.3(16.2-34.4)$ & 6.470 & 0.011 \\
\hline II/III & $13.2(9.9-16.4)$ & & \\
\hline \multicolumn{4}{|l|}{ CA19-9 (U/mL) } \\
\hline$<37$ & $38.0(6.1-69.9)$ & 6.911 & 0.009 \\
\hline$\geq 37$ & $14.7(12.5-16.8)$ & & \\
\hline \multicolumn{4}{|l|}{ Cbl-b expression } \\
\hline Low & $24.0(17.1-30.9)$ & 6.167 & 0.013 \\
\hline High & $13.9(10.7-17.0)$ & & \\
\hline
\end{tabular}

pT: pathological primary tumor; pN: pathological regional lymph nodes; pTNM: pathological TNM. 
Table 3: Multivariate analysis of prognostic factors independently associated with overall survival (OS) in patients with pancreatic ductal adenocarcinoma treated by surgical resection

\begin{tabular}{lcccc}
\hline Characteristics & Category & Hazard ratio & 95\% CI & P value \\
\hline Cbl-b expression & high vs. low & 2.048 & $1.285-3.266$ & 0.003 \\
CA19-9 (U/mL) & $\geq 37$ vs. $<37$ & 2.765 & $1.424-5.369$ & 0.003 \\
pN category & Yes vs. No & 1.713 & $1.112-2.638$ & 0.015 \\
Histological differentiation & poor vs. well/moderate & 2.299 & $1.354-3.905$ & 0.002 \\
\hline
\end{tabular}

The multivariate Cox proportional hazards model (forward selection) was fitted using all of the clinical and pathological variables, which included age, gender, maximal tumor diameter, histological differentiation, surgical margins, pT category, pN category, vascular tumor thrombus, invasion of adjacent organs, pTNM stage, serum CA19-9 levels, and tissue Cbl-b expressions.

\section{Univariate and multivariate analysis of prognostic factors for overall survival}

The log-rank test results are shown in Table 2. Age $(P=0.049)$, histological differentiation $(P=0.013), \mathrm{pN}$ category $(P=0.009)$, pTNM stage $(P=0.011)$, serum CA19-9 level $(P=0.009)$, and Cbl-b expression $(P=$ 0.013 ) were significantly associated with OS. In univariate analysis, other characteristics (sex, maximum tumor diameter, surgical margins, pT category, vascular tumor thrombus, invasion of adjacent organs) did not influence OS (all $P>0.05$ ). The multivariate Cox proportional hazards model (forward selection) was fitted using all 12 clinical and pathological variables, and found that high Cbl-b expression (HR, 2.048; 95\% CI: 1.285-3.266; $P=0.003$ ), serum CA19-9 level $\geq 37 \mathrm{U} / \mathrm{mL}$ (HR, 2.765;
A

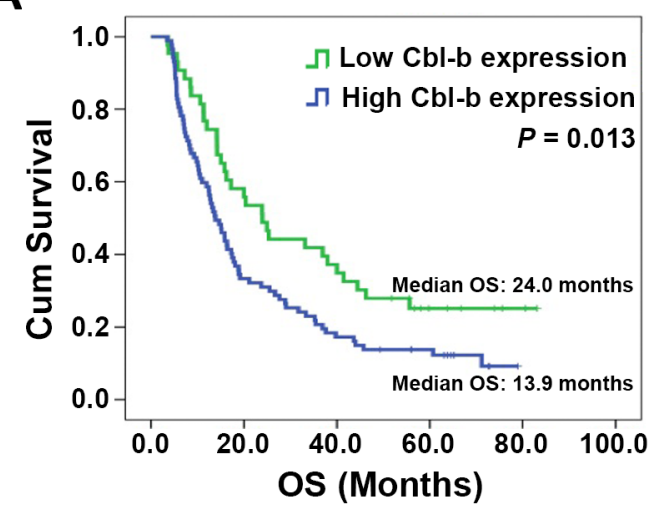

C

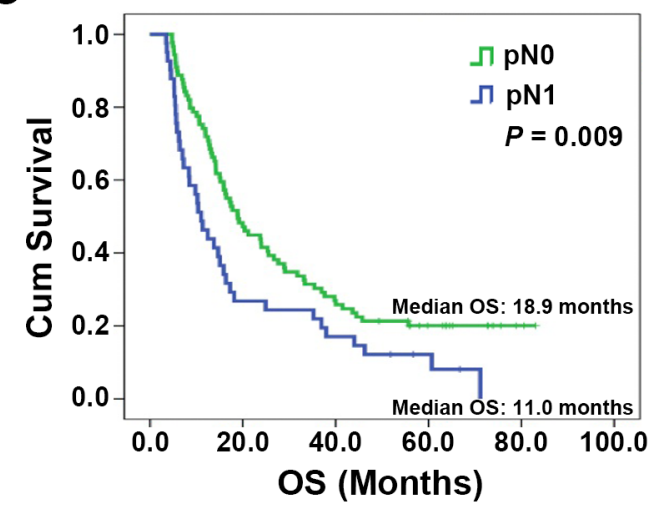

B

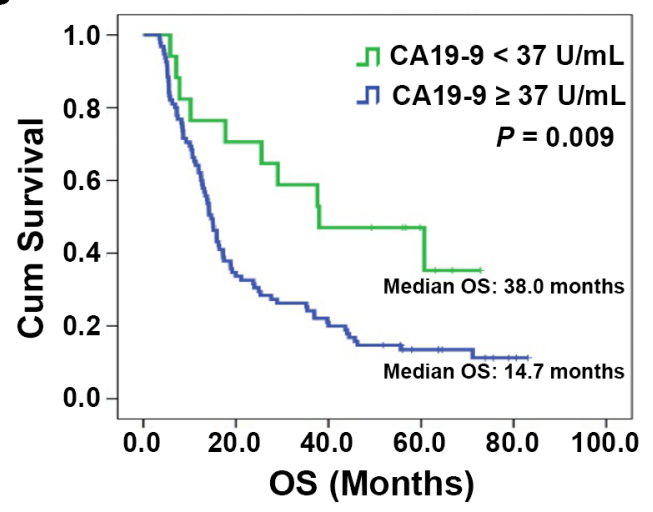

D

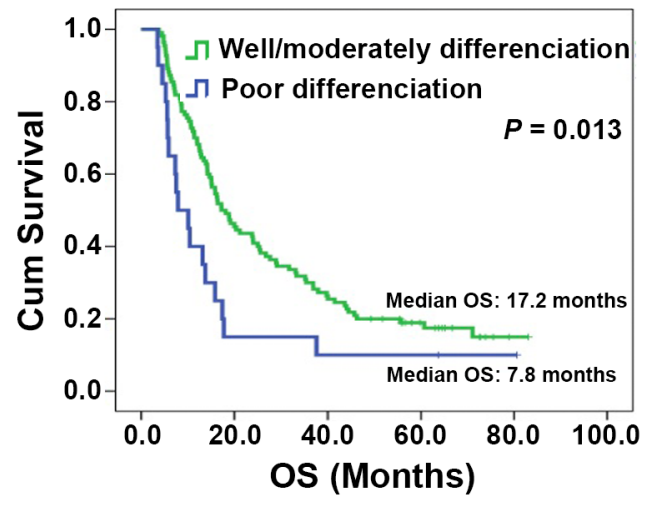

Figure 2: Overall survival (OS) in patients stratified by prognostic factors found to be independently associated with OS. ((A) Cbl-b expression; (B) Serum CA19-9 levels; (C) pN category; (D) Histological differentiation). Significant differences in OS were revealed by the log-rank test. 
Table 4: Comparison of the prognostic accuracies of different models

\begin{tabular}{lcc}
\hline Model & C-index & AIC \\
\hline Cbl-b & 0.572 & 751.641 \\
$\mathrm{pN}+$ Differentiation + CA19-9 & 0.666 & 741.623 \\
Cbl-b + CA19-9 + pN + Differentiation & 0.680 & 732.959 \\
\hline
\end{tabular}

pN: pathological regional lymph nodes; C-index: Harrell's concordance index; AIC: Akaike information criterion.

95\% CI: 1.424-5.369; $P=0.003)$, lymph node metastasis (HR, 1.713; 95\% CI: $1.112-2.638 ; P=0.015)$, and poor tumor differentiation (HR, 2.299; 95\% CI: 1.354-3.905; $P=0.002)$ were independently associated with the poor OS (Table 3). As shown in Figure 2, patients with increased Cbl-b expression, increased CA19-9 level, lymph node metastasis, or poor tumor differentiation had shorter OS and that the OS in sub-group of serum CA19-9 $\geq 37 \mathrm{U} / \mathrm{mL}$ was significantly associated with Cbl-b expression (Figure 3 ). These data suggested that, in addition to currently used markers ( $\mathrm{pN}$ category, CA19-9 level, and histological differentiation), Cbl-b has prognostic value in patients with resectable PDAC.

\section{Predictive nomograms for OS}

To develop an intuitive and quantitative method of stratifying patients by prognosis, nomograms predicting 1- and 3-year OS were developed from the statistical models (Figure 4A). The predictors, including Cbl-b expression, $\mathrm{pN}$ category, histological differentiation, and CA19-9 level, were all independently associated with OS in multivariable analysis. Each predictor in the nomogram was weighted a number of points, and the total points for each patients was in accordance with a special predicted 1- and 3-year OS. Higher scores indicated worse prognosis (Figure 4A). The model showed good accuracy

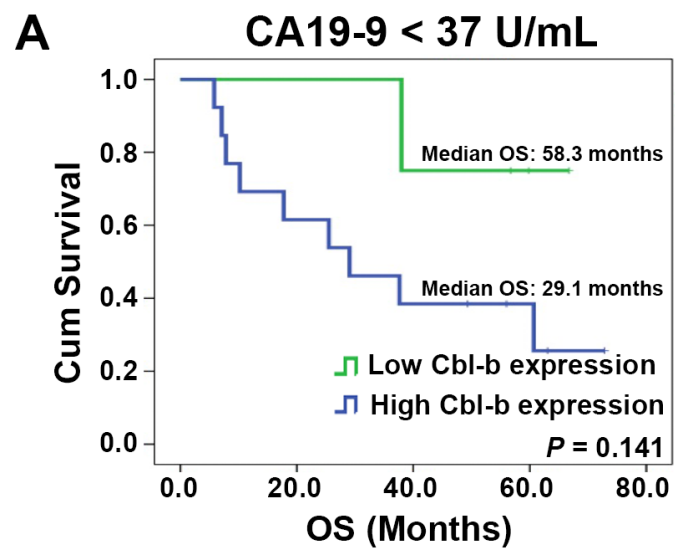

for predicting overall survival rate of PDAC treated by surgical resection, with a Harrell's concordance index (C-index) of 0.680. Calibration curves for the nomogram predicted 1-year and 3-year OS consistent with the ideal model (Figure 4B-4C). The patients were divided into three subgroups according to the tertiles of the scores calculated by the prognostic nomogram. Kaplan-Meier curves for the OS showed that each group had a different prognosis (log-rank $x^{2}=16.596, P<0.001$ ) (Figure 4D).

In addition, to investigate whether the combination of Cbl-b expression with the traditional variables (including $\mathrm{pN}$ category, histological differentiation and CA19-9 level) could improve the predictive accuracy for PDAC, the $\mathrm{C}$-index or Akaike information criterion (AIC) of each predictive model was compared. As shown in Table 4, the C-index of Cbl-b expression model and the traditional variables model was 0.572 and 0.666 , respectively, but improved to 0.680 when Cbl-b expression was combined with the traditional variables. Similarly, the AIC of Cbl-b expression model and the traditional variables model was 751.641 and 741.623 , respectively, and decreased to 732.959 when the Cbl-b expression was combined with the traditional variables. The data indicated that the combination of Cbl-b expression with pN category, histological differentiation and CA19-9 level could achieve a more reliable and precise prognostic prediction for OS of resectable PDAC patients.

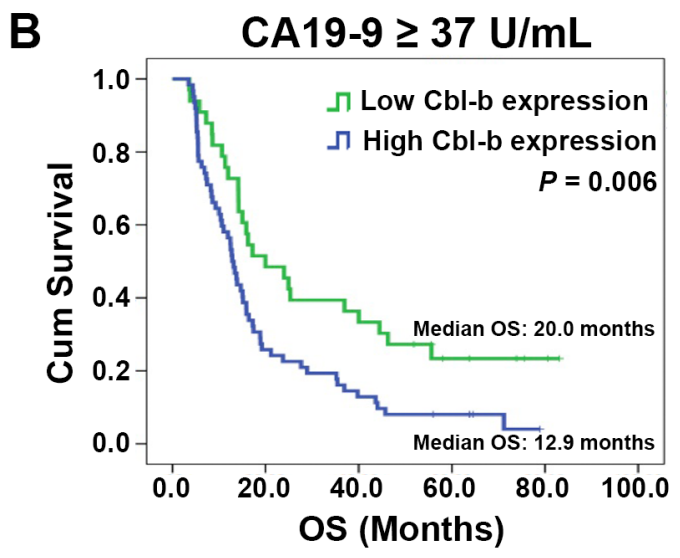

Figure 3: Overall survival (OS) in patients with different CA19-9 levels according to Cbl-b expression. ((A) CA19-9 <37 $\mathrm{U} / \mathrm{mL}$; (B) CA19-9 $\geq 37 \mathrm{U} / \mathrm{mL}$ ). Significant differences were revealed by the log-rank test. 


\section{DISCUSSION}

To date, various clinicopathological features and biological markers have been reported to be prognostic factors in patients with resectable PDAC, including histological differentiation, $\mathrm{pN}$ category, and serum CA199 level [6, 18-20]. All are useful for guiding standard clinical management and establishing individualized treatment plans. In a previous study, we found that silencing of Cbl-b expression inhibited proliferation in PDAC cells in vitro [9]. In this study, we investigated the prognostic value of Cbl-b by retrospectively evaluating the relationship of Cbl-b expression with the clinicopathological characteristics and survival of PDAC patients. A statistically significant association was found between Cbl-b expression and survival, showing that Cbl-b level has prognostic value in patients with resectable PDAC.

Cbl-b consists of an N-terminal tyrosine kinase binding (TKB) domain, linker, RING finger domain,
C-terminal proline-rich region, ubiquitin associated domain, and a leucine zipper region [21]. The specificity of Cbl-b binding to substrate proteins is determined by the recognition of specific phosphorylated tyrosine residues by the TKB domain. The RING finger has intrinsic E3 ligase activity and mediates the transfer of ubiquitin to substrates. Therefore, Cbl-b has dual functions as a ubiquitin-protein ligase and an adaptor protein $[12,22$, 23]. In the immune system, Cbl-b can inhibit activation of both $\mathrm{T}$ CD8+ cells and natural killer (NK) cells, thereby inhibiting the inherent antitumor immune response and accelerating tumor development and progression [24]. The genetic deletion or functional inactivation of Cbl-b in NK cells can significantly inhibit the proliferation and metastasis of melanomas [25], and knockout of c-Cbl, a homologue of Cbl-b, can inhibit the proliferation of prostate cancer cells [26]. Cbl-b has also been shown to promote the proliferation of breast cancer cells [27]. We previously showed that knockdown of Cbl-b significantly decreased the proliferative activity of PDAC cells and that

A

Points

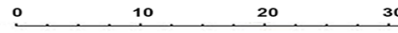

30 . 40

50

60

High

CbI-b

Low

PN1

Histological differentiation

CA19-9

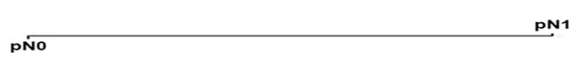

Total Points$$
0
$$

1-Year Survival

3-Year Survival
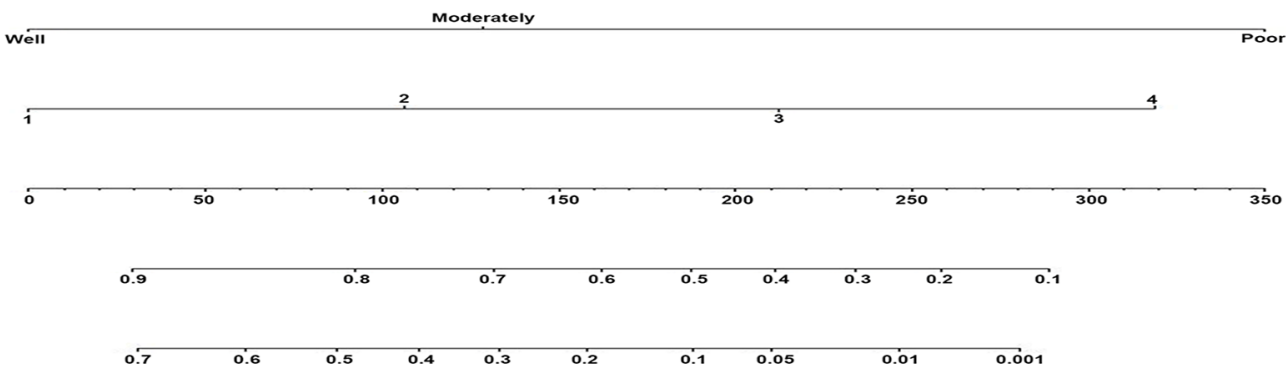

B

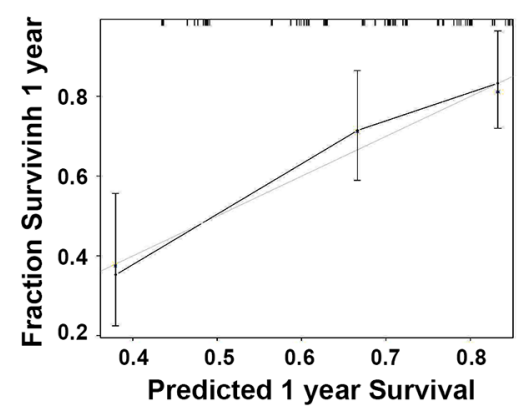

C

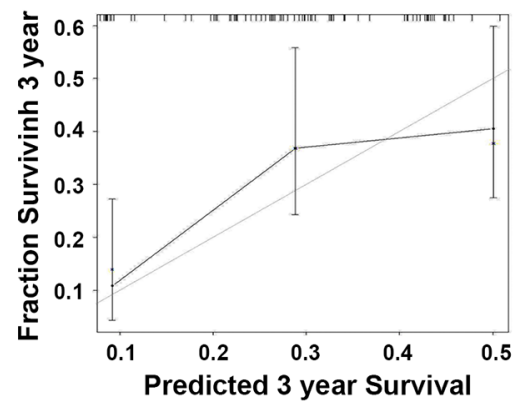

D

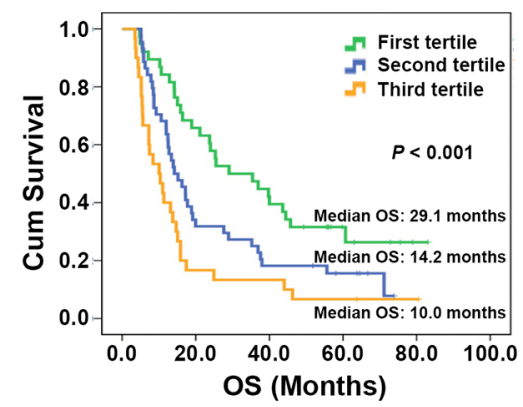

Figure 4: Prognostic nomogram for predicting overall survival (OS) in patients with pancreatic ductal adenocarcinoma (PDAC) treated by surgical resection. Prognostic nomogram (A) and calibration curve (B-C) of Cbl-b expression combined with histological differentiation, $\mathrm{pN}$ category, and serum CA19-9 levels in patients with resectable PDAC. *For the nomogram, the values of CA19-9 were devided into 4 groups: CA19-9 $<37 \mathrm{U} / \mathrm{mL}$; the values of CA19-9 $\geq 37 \mathrm{U} / \mathrm{mL}$ was also divided into another 3 groups: the CA19-9 values were sorted from low to high, and the values of 33\% and 67\% were used as the cutoff points. (D) Kaplan-Meier curves for the OS in patients grouped according to the tertiles of the scores calculated by the prognostic nomogram. Each group represented a different prognosis (log-rank $\left.x^{2}=16.596, P<0.001\right)$. 
expression of Cbl-b and Ki-67 protein were positively correlated in PDAC tissue [9]. In this study, univariate and multivariate analysis found that Cbl-b expression had prognostic value, and was independently associated with OS of resectable PDACs.

The function of Cbl-b in tumor cells is controversial. As a negative regulator of growth factor receptor signaling and the suppression of cancer cell proliferation, Cbl-b is regarded as having anti-tumor activity. 32D/EGFR cells overexpressing Cbl-b have a markedly reduced proliferative response to epidermal growth factor (EGF), and an increased apoptosis rate [28]. Cbl-b knockdown could promote cell proliferation and reduce apoptosis induced by 5 -fluorouracil treatment in gastric cancer cells via the EGFR pathway [29]. Other studies have also indicated that $\mathrm{Cbl}-\mathrm{b}$ promotes the proliferation of cancer cells. In breast cancer, Cbl-b binds to the proline rich region of Smad3 and prevents the protein from translocating into the nucleus to inhibit the transcription of tumor suppressor genes downstream of the TGF- $\beta$ pathway, including $\mathrm{p} 21^{\mathrm{Cip} 1}$ and $\mathrm{p} 15^{\mathrm{INK} 4 \mathrm{~b}}$ [27]. We previously showed that silencing of Cbl-b expression inhibited proliferation in PDAC cells by up-regulation of Smad3/p21 signaling [9]. The diversity of Cbl-b substrates in cancer cells and the regulation of pathways in a cell type-dependent manner may account for differences in the effect of Cbl-b expression on proliferation of cancer cells.

This study demonstrated that serum CA19-9 levels $\geq 37 \mathrm{U} / \mathrm{mL}$, poor histological differentiation, and lymph node metastasis was significantly associated with poor OS, which is consistent with previous studies [6, 18-20]. Cbl-b had significant prognostic value in a subgroup of patients with serum CA19-9 $\geq 37 \mathrm{U} / \mathrm{mL}$, indicating shorter OS in patients with serum CA19-9 $\geq 37 \mathrm{U} / \mathrm{mL}$ and tumors with high Cbl-b expression. These findings suggested that combination Cbl-b expressions and CA19-9 levels might provide more information for prognostic prediction of PDACs. For the sub-group of serum CA19-9 $<37$ U/ $\mathrm{mL}$, the median OS of patients with Cbl-b low expression was longer than that with Cbl-b high expression, but no statistical significance was observed $(P=0.141)$. It is probably because that there were only 18 patients in the low CA19-9 group vs. 98 in the high CA19-9 group. The groups were very different in size and therefore it is difficult to draw conclusions from the low CA19-9 group. Prospective validation, including an adequate number of patients, is required to clarify the relationship between the CA19-9 level and Cbl-b expression in PDAC. Nomograms and calibration graphs had a C-index of 0.680 , which supports the value of Cbl-b to predict 1 -year and 3-year survival rates. Nevertheless, this study had limitations much like those of other retrospective studies. Consequently, results require validation in a large, prospective, multicenter randomized trial.

In conclusion, Cbl-b expression clearly indicated unfavorable prognosis and might be adopted as a novel prognostic marker in patients with resectable PDAC. Combining Cbl-b expression with serum CA19-9, pN category, and histological differentiation gave more precise prognostic information in PDAC patients, and might help to identify patients in need of much more stringent postoperative follow up. Detecting Cbl-b expression might assist clinicians in the choice of optimal treatment and postoperative management of PDAC patients.

\section{MATERIALS AND METHODS}

\section{Patients and tissue samples}

A total of 182 consecutive patients with resectable primary pancreatic carcinoma were treated at the Shengjing Hospital of China Medical University between January 2009 and February 2012. The diagnosis was confirmed by histopathology. Patients with intraductal papillary mucinous adenocarcinoma, mucinous carcinoma, malignant endocrine tumors, adenosquamous carcinoma, squamous carcinoma, and acinar cell carcinoma were excluded. Patients with a second cancer with a life-threatening phenotype (e.g., gastric stromal tumor, renal clear cell carcinoma, adrenocortical adenocarcinoma, gallbladder adenocarcinoma, and ovarian mucinous cystadenocarcinoma), patients died in the hospital within 30 days after surgery, and those with incomplete clinicopathological data were also excluded. The remaining 134 patients with PDAC were included in the study. None had received chemotherapy or radiation therapy prior to surgery. All patient data were retrospectively reviewed after the Research Ethics Committee of Shengjing Hospital of China Medical University approved the study.

Patient age, sex, location of the tumor, type of resection, tumor size, histologic differentiation, margin status (pancreatic resection, biliary, posterior, retroperitoneal, and mesenteric margins), tumor stage, node stage, vessel invasion, vascular tumor thrombus, invasion of adjacent organs (bile duct, duodenum, stomach, colon, jejunum, and spleen), tumor-nodemetastasis (TNM) stage according to the seventh American Joint Committee on Cancer (AJCC) TNM system criteria, and preoperative serum CA19-9 level. Patients or their relatives and clinicians were followed up until 31 March 2016 by telephone interview to monitor survival or document the day of death. Death was confirmed from official local government death certificates or medical agencies, or by information obtained from family members. The medical records were independently reviewed by a physician to confirm the cause of death. A standard regimen of intravenous gemcitabine at a conventional dose and schedule was administered as postoperative adjuvant chemotherapy to patients who were willing and able to tolerate it, regardless of margin status or tumor stage. 


\section{Immunohistochemistry (IHC)}

PDAC tissue sections were deparaffinized, rehydrated, and washed in phosphate buffered saline (PBS) using an S-P immunohistochemistry kit (Fuzhou Maixin Biological Technology Ltd., Fujian, China) following the manufacturer's instructions. Then slides were incubated with mouse anti-human Cbl-b (Santa Cruz Biotechnology, CA, USA) primary antibody in PBS at $4^{\circ} \mathrm{C}$ overnight in a humid box. After washing in PBS, the slides were incubated with secondary antibody, and the immune complexes were stained with 3,30-diaminobenzidine tetrahydrochloride (DAB; Fuzhou Maixin Biological Technology Ltd., Fujian, China) following the kit manufacturer's instructions. Finally, the slides were counterstained with hematoxylin. Pre-immune rabbit serum at the same dilution was used as a negative control. Slide was evaluated by scanning the entire tissue specimen at low magnification $(\times 10)$ and staining was confirmed at high magnification $(\times 20$ and $\times 40)$. Tumors with $<10 \%$ Cbl-b stained cells were considered low expression; those with $\geq 10 \%$ Cbl-b-stained cells were considered high expression. Two pathologists independently scored the slides.

\section{Statistical analysis}

Data analysis was performed using SPSS software version 13.0 (SPSS Inc., Chicago, IL, USA) and R 3.3.1 software (http://www.R-project.org). Categorical variables were reported as numbers and percentages. Median values were used as cutoffs to categorize patient clinicopathological variables, and comparisons of Cbl-b expression were performed with the two-tailed $x^{2}$ or Fisher's exact test. The upper limit of the normal reference value $(37 \mathrm{U} / \mathrm{mL})$ was used as the cutoff for CA19-9; and for the nomogram, the group of CA19-9 $\geq 37 \mathrm{U} / \mathrm{mL}$ was also divided into another 3 groups: the CA19-9 values were sorted from low to high, and the values of $33 \%$ and $67 \%$ were used as the cutoff points. Overall survival (OS) was defined as the interval between the date of the surgery and either the date of death from any cause or the last follow-up visit. Survival curves were calculated by the Kaplan-Meier method, and the significance of differences in survival were determined by the log-rank test. A multivariate Cox proportional hazards model (forward selection) was fitted using all clinical and pathological variables, and risk factors identified in the multivariate analysis were used to construct a nomogram. The C-index and the AIC were used to evaluate the discrimination of the nomogram to predict prognosis. The higher the $\mathrm{C}$-index, or the lower the AIC, the more accuracy was the prognostic prediction. Calibration curves were drawn to compare the relationship between observed and predicted responses. Two-sided $P$-values $<0.05$ were considered statistically significant.

\section{Abbreviations}

Cbl-b: Casitas B-lineage lymphoma b; PDAC: pancreatic ductal adenocarcinoma; pT: pathological primary tumor; pTNM: pathological TNM; pN: pathological regional lymph node; OS: overall survival; CA19-9: carbohydrate antigen 19-9; CI: confidence interval; C-index: Harrell's concordance index; AIC: Akaike information criterion; TKB: tyrosine kinase binding; NK: natural killer; EGF: epidermal growth factor; TNM: tumor-node-metastasis; AJCC: American Joint Committee on Cancer; IHC: immunohistochemistry; PBS: phosphate buffered saline; C-index: concordance index.

\section{Author contributions}

Qian Dong carried out the records collected and analyzed, performed the experiments, and drafted the manuscript. Yuteng Ma, Yao Zhang and Yafei Qi participated in records collected. Xianghong Yang and Xiaofang Che participated in the design of the study. Zhi Li and Ce Li participated in the statistical analysis. Xiujuan Qu, Yunpeng Liu and Kai Li conceived of the study, and participated in its design and coordination. All authors read and approved the final manuscript.

\section{ACKNOWLEDGMENTS}

The authors thank Liqiang Zheng for useful discussion, and Fang Dong and Ying Zhang for kindly providing technical support.

\section{CONFLICTS OF INTEREST}

The authors disclose no potential conflicts of interest.

\section{GRANT SUPPORT}

This work was supported by the Outstanding Scientific Fund of Shengjing Hospital (No. 201210), the Science and Technology Project Fund from Education Department of Liaoning Province (Nos. LK201619, L2015599), the Science and Technology Plan Project of Liaoning Province (Nos. 2014226033, 2014225013), and the general project of Liaoning Province Department of Education (No. LS201613), Project for clinical ability construction of Chinese medicine.

\section{REFERENCES}

1. Waddell N, Pajic M, Patch AM, Chang DK, Kassahn KS, Bailey P, Johns AL, Miller D, Nones K, Quek K, Quinn MC, Robertson AJ, Fadlullah MZ, et al. Whole genomes redefine the mutational landscape of pancreatic cancer. Nature. 2015; 518:495-501. 
2. Luo J, Xiao L, Wu C, Zheng Y, Zhao N. The incidence and survival rate of population-based pancreatic cancer patients: Shanghai Cancer Registry 2004-2009. PLoS One. 2013; 8:e76052.

3. Pannala R, Basu A, Petersen GM, Chari ST. New-onset diabetes: a potential clue to the early diagnosis of pancreatic cancer. Lancet Oncol. 2009; 10:88-95.

4. Winter JM, Cameron JL, Campbell KA, Arnold MA, Chang DC, Coleman J, Hodgin MB, Sauter PK, Hruban RH, Riall TS, Schulick RD, Choti MA, Lillemoe KD, et al. 1423 pancreaticoduodenectomies for pancreatic cancer: a singleinstitution experience. J Gastrointest Surg. 2006; 10:11991210; discussion 1210-1191.

5. Goonetilleke KS, Siriwardena AK. Systematic review of carbohydrate antigen (CA 19-9) as a biochemical marker in the diagnosis of pancreatic cancer. Eur J Surg Oncol. 2007; 33:266-270.

6. Hartwig W, Strobel O, Hinz U, Fritz S, Hackert T, Roth C, Buchler MW, Werner J. CA19-9 in potentially resectable pancreatic cancer: perspective to adjust surgical and perioperative therapy. Ann Surg Oncol. 2013; 20:2188-2196.

7. Sugiura T, Uesaka K, Kanemoto H, Mizuno T, Sasaki K, Furukawa H, Matsunaga K, Maeda A. Serum CA19-9 is a significant predictor among preoperative parameters for early recurrence after resection of pancreatic adenocarcinoma. J Gastrointest Surg. 2012; 16:977-985.

8. Wang Y, Lin J, Cui J, Han T, Jiao F, Meng Z, Wang L. Prognostic value and clinicopathological features of PD-1/PD-L1 expression with mismatch repair status and desmoplastic stroma in Chinese patients with pancreatic cancer. Oncotarget. 2017; 8:9354-9365. doi: 10.18632/ oncotarget.14069.

9. Dong Q, Li C, Che X, Qu J, Fan Y, Li X, Li Y, Wang Q, Liu Y, Yang X, Qu X. MicroRNA-891b is an independent prognostic factor of pancreatic cancer by targeting Cbl-b to suppress the growth of pancreatic cancer cells. Oncotarget. 2016; 7:82338-82353. doi: 10.18632/oncotarget.11001.

10. Pietrasz D, Pecuchet N, Garlan F, Didelot A, Dubreuil O, Doat S, Imbert-Bismut F, Karoui M, Vaillant JC, Taly V, Laurent-Puig P, Bachet JB. Plasma circulating tumor DNA in pancreatic cancer patients is a prognostic marker. Clin Cancer Res. 2017; 23:116-123.

11. Liu Q, Zhou H, Langdon WY, Zhang J. E3 ubiquitin ligase Cbl-b in innate and adaptive immunity. Cell Cycle. 2014; 13:1875-1884.

12. Liyasova MS, Ma K, Lipkowitz S. Molecular pathways: cbl proteins in tumorigenesis and antitumor immunityopportunities for cancer treatment. Clin Cancer Res. 2015; 21:1789-1794.

13. Zhang L, Teng Y, Fan Y, Wang Y, Li W, Shi J, Ma Y, Li C, Shi X, Qu X, Liu Y. The E3 ubiquitin ligase Cbl-b improves the prognosis of RANK positive breast cancer patients by inhibiting RANKL-induced cell migration and metastasis.
Oncotarget. 2015; 6:22918-22933. doi: 10.18632/ oncotarget.4382.

14. Oguro S, Ino Y, Shimada K, Hatanaka Y, Matsuno Y, Esaki M, Nara S, Kishi Y, Kosuge T, Hiraoka N. Clinical significance of tumor-infiltrating immune cells focusing on BTLA and Cbl-b in patients with gallbladder cancer. Cancer Sci. 2015; 106:1750-1760.

15. Zhang Y, Qu X, Teng Y, Li Z, Xu L, Liu J, Ma Y, Fan Y, Li C, Liu S, Wang Z, Hu X, Zhang J, et al. Cbl-b inhibits P-gp transporter function by preventing its translocation into caveolae in multiple drug-resistant gastric and breast cancers. Oncotarget. 2015; 6:6737-6748. doi: 10.18632/ oncotarget. 3253.

16. Li P, Wang X, Liu Z, Liu H, Xu T, Wang H, Gomez DR, Nguyen QN, Wang LE, Teng Y, Song Y, Komaki R, Welsh JW, et al. Single nucleotide polymorphisms in CBLB, a regulator of T-cell response, predict radiation pneumonitis and outcomes after definitive radiotherapy for nonsmall-cell lung cancer. Clin Lung Cancer. 2016; 17:253262. e255.

17. Makishima H, Cazzolli H, Szpurka H, Dunbar A, Tiu R, Huh J, Muramatsu H, O'Keefe C, Hsi E, Paquette RL, Kojima S, List AF, Sekeres MA, et al. Mutations of e3 ubiquitin ligase cbl family members constitute a novel common pathogenic lesion in myeloid malignancies. J Clin Oncol. 2009; 27:6109-6116.

18. Dong Q, Yang XH, Zhang Y, Jing W, Zheng LQ, Liu YP, Qu XJ. Elevated serum CA19-9 level is a promising predictor for poor prognosis in patients with resectable pancreatic ductal adenocarcinoma: a pilot study. World J Surg Oncol. $2014 ; 12: 171$.

19. Dong Q, Zhang Y, Yang XH, Jing W, Zheng LQ, Liu YP, Qu XJ, Li Z. Serum calcium level used as a prognostic predictor in patients with resectable pancreatic ductal adenocarcinoma. Clin Res Hepatol Gastroenterol. 2014; 38:639-648.

20. Strobel O, Hinz U, Gluth A, Hank T, Hackert T, Bergmann F, Werner J, Buchler MW. Pancreatic adenocarcinoma: number of positive nodes allows to distinguish several $\mathrm{N}$ categories. Ann Surg. 2015; 261:961-969.

21. Lutz-Nicoladoni C, Wolf D, Sopper S. Modulation of immune cell functions by the E3 ligase Cbl-b. Front Oncol. $2015 ; 5: 58$.

22. Thien CB, Langdon WY. c-Cbl and Cbl-b ubiquitin ligases: substrate diversity and the negative regulation of signalling responses. Biochem J. 2005; 391:153-166.

23. Krawczyk C, Bachmaier K, Sasaki T, Jones RG, Snapper SB, Bouchard D, Kozieradzki I, Ohashi PS, Alt FW, Penninger JM. Cbl-b is a negative regulator of receptor clustering and raft aggregation in T cells. Immunity. 2000; 13:463-473.

24. Matalon O, Barda-Saad M. Cbl ubiquitin ligases mediate the inhibition of natural killer cell activity. Commun Integr Biol. 2016; 9:e1216739. 
25. Paolino M, Choidas A, Wallner S, Pranjic B, Uribesalgo I, Loeser S, Jamieson AM, Langdon WY, Ikeda F, Fededa JP, Cronin SJ, Nitsch R, Schultz-Fademrecht C, et al. The E3 ligase Cbl-b and TAM receptors regulate cancer metastasis via natural killer cells. Nature. 2014; 507:508-512.

26. Knight JF, Shepherd CJ, Rizzo S, Brewer D, Jhavar S, Dodson AR, Cooper CS, Eeles R, Falconer A, Kovacs G, Garrett MD, Norman AR, Shipley J, et al. TEAD1 and c-Cbl are novel prostate basal cell markers that correlate with poor clinical outcome in prostate cancer. Br J Cancer. 2008; 99:1849-1858.
27. Kang JM, Park S, Kim SJ, Hong HY, Jeong J, Kim HS, Kim SJ. CBL enhances breast tumor formation by inhibiting tumor suppressive activity of TGF-beta signaling. Oncogene. 2012; 31:5123-5131.

28. Ettenberg SA, Keane MM, Nau MM, Frankel M, Wang LM, Pierce JH, Lipkowitz S. cbl-b inhibits epidermal growth factor receptor signaling. Oncogene. 1999; 18:1855-1866.

29. Feng D, Ma Y, Liu J, Xu L, Zhang Y, Qu J, Liu Y, Qu X. Cbl-b enhances sensitivity to 5-fluorouracil via EGFR- and mitochondria-mediated pathways in gastric cancer cells. Int J Mol Sci. 2013; 14:24399-24411. 